\title{
Suppression of Vortex Precession in a Non-Contact Handling Device by a Circular Column
}

\author{
Shouichiro Iio, Katsuya Hayashi, Eri Akahane, Yusuke Katayama, Salisa Veerapun, Xin Li, \\ Toshiharu Kagawa \\ Department of Environmental Science and Technology, Shinshu University, Nagano, Japan \\ Email: shouiio@shinshu-u.ac.jp
}

Received 4 December 2015; accepted 22 March 2016; published 29 April 2016

Copyright (C) 2016 by authors and Scientific Research Publishing Inc.

This work is licensed under the Creative Commons Attribution International License (CC BY). http://creativecommons.org/licenses/by/4.0/

(c) (i) Open Access

\begin{abstract}
Vortex levitation attains non-contact handling by injecting air through a tangential nozzle into a cylindrical cup generating the swirling flow. The precessing of the swirling flow causes pressure fluctuation. This phenomenon becomes apparent as the gap between the cup and workpiece increases, which significantly disturbs the stability of conveyance. In this paper, suppression of pressure fluctuation by a cylindrical column that stabilizes the vortex levitation is described and its mechanism is mentioned. According to the experimental set up, the pressure was measured at the center of the workpiece and the wall of the cup; velocity field under the work piece was visualized by PIV. The result suggested that the larger diameter column denoted the effect on suppression of the fluctuation because the precessing of the swirling flow became stable. On the other hand, variation of the column thickness had insignificant effect on suppressing the fluctuation, but sucking force became weakened since the swirling velocity decreased.
\end{abstract}

\section{Keywords}

Non-Contact Handling, Swirling Flow, Pressure Fluctuation, Visualization

\section{Introduction}

In semiconductor manufacturing, a direct contact handling method is used to convey a workpiece: wafers or glass substrates. However, direct contact between the workpiece and the handling device often leads to surface scratching, static electricity and particle contamination. Non-contact and stable handling methods are preferred to improve production quality and efficiency. The thinner and larger the workpiece is, the higher the necessity of 
the non-contact handling devices is. A number of non-contact handling methods have been proposed so far, many of which demonstrated effectiveness. Among those, the prominent methods are magnetic, electrostatic and pneumatic [1] [2]. The magnetic and the electrostatic methods have restricted usage to conductive materials, whereas the pneumatic methods have not; it is applicable to a wide range of materials including: insulator or conductor, magnetic or non-magnetic, rigid or non-rigid and so on because the handling force produced by the air flow is magnetic-free and low-heat-generate [2] [3]. Because of its simple structure and operation, the pneumatic method provides minimal maintenance and does not require a control loop to obtain a stable state. The most popular pneumatic method is Bernoulli levitation based on Bernoulli principle [3]-[5]. However, Bernoulli levitation needs high air consumption against the sucking power and often leads to a high air power loss through supply pipes [3]. Therefore, the vortex levitation, which is one of the latest pneumatic non-contact handling methods, is proposed here.

In this levitation, a vortex cup is used to generate the air swirling flow. As presented in Figure 1, the cup composes of a circular cylinder and a tangential nozzle. Compressed air is injected through the nozzle, circulated along the circular wall, and generates swirling flow. The negative pressure in the central area of the swirling flow generates vacuum pressure. Since the working fluid is supplied continuously, the workpiece is kept levitating at certain distance away from the cup at equilibrium where the weight of the workpiece balances the sucking force. Figure 2 shows the image of a manipulator in practical use [12]. It is equipped with six vortex cups providing better stability and larger sucking force. A number of previous studies have reported the characteristics of the swirling flow in cylindrical chambers and circular pipes [6]-[8], and their technical applications include separation of particles by cyclones and improvement of combustion by swirl burners, among others [9]-[11]. Unfortunately, these applications cannot be applied directly to the vortex cup because one side of the cup is opened. When the gap between the workpiece and the cup increases, the vortex core precesses, and periodic pressure fluctuation is then generated [12]-[15]. This fluctuation disturbs stable handling of the workpiece. The theoretical approach for indirectly estimating the pressure distribution in the cup was conducted by Li et al. [16]. It is not only important but also necessary to develop suppression method of the pressure fluctuation. This study, therefore, focused on suppression of the fluctuation using a circular column. The interest was paid to investigate the influence of the height and diameter of the column with visualization and pressure measurement.

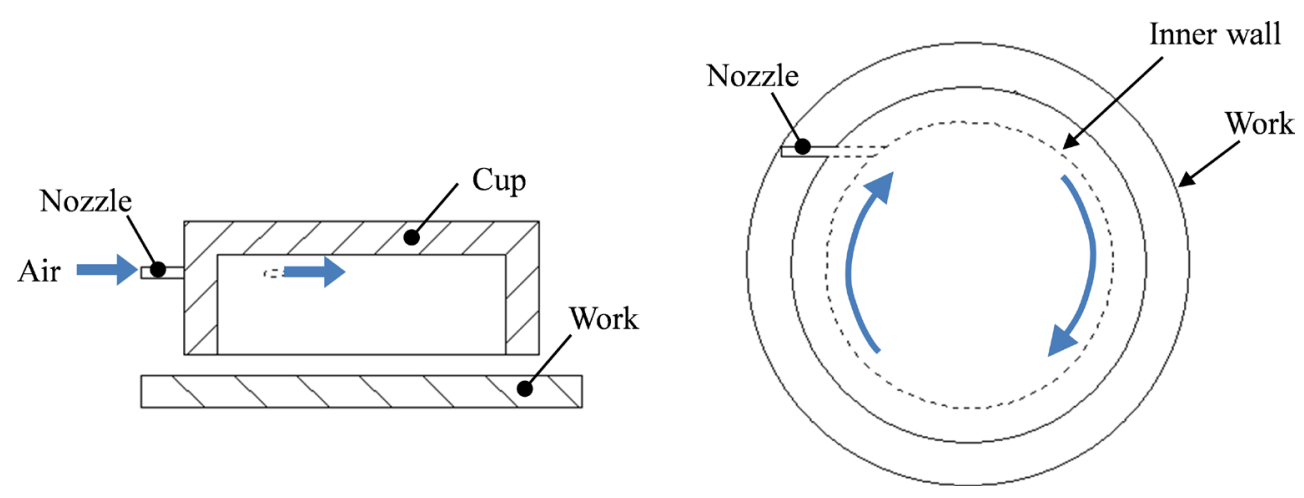

Figure 1. Vortex cup.

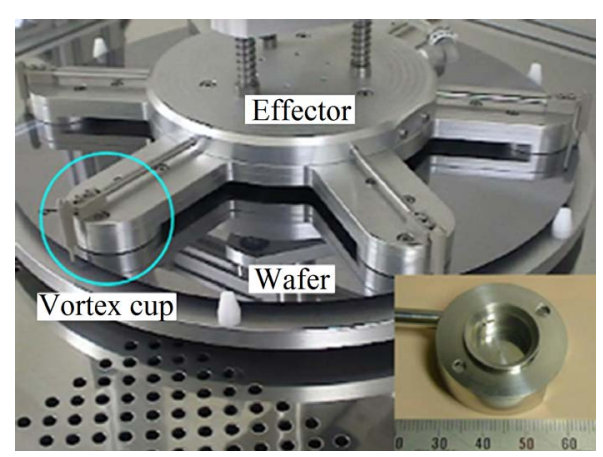

Figure 2. Non-contact handling system using vortex cups. [12]. 


\section{Experimental Apparatus and Procedures}

In the vortex levitation, a vortex cup is used to generate the air swirling flow. Sucking pressure is generated by injecting air into the vortex cup through the tangential nozzle. The maximum swirling velocity reaches approximately $50 \mathrm{~m} / \mathrm{s}$. The flow field is the most important to the vortex cup performance, but its behavior is difficult to grasp due to the resolution of space and time. In order to get a better visualization of the flow behavior, water was selected as a working fluid instead of air, and the vortex cup was scaled up. Figure 3 illustrates the experimental apparatus consisting of; a head tank, a test section tank and a vortex cup. The head tank and the test section tank have an overflow system to maintain constant flow rate. Water in the head tank enters the cup through a water pipe. The flow rate is controlled by hand valves and measured by a weighting method. Figure 4 illu-

Head tank

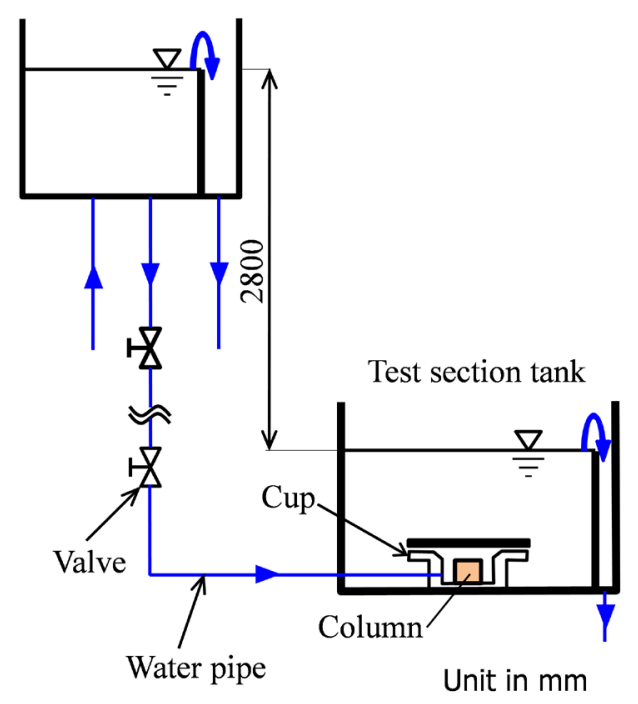

Figure 3. Experimental apparatus.

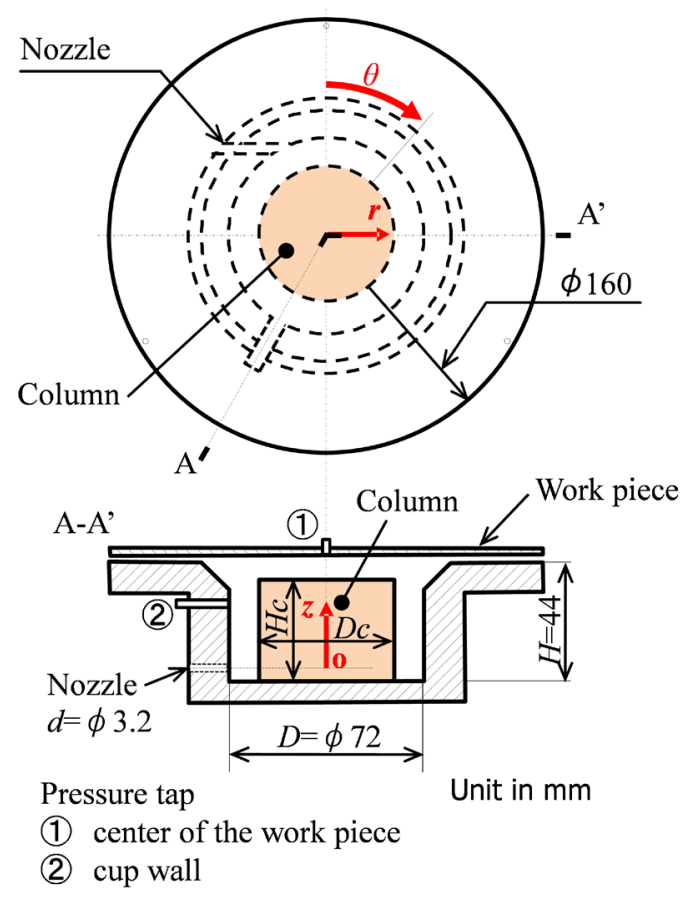

Figure 4. Test section. 
strates the cup configuration: $D=72 \mathrm{~mm}, H=44 \mathrm{~mm}, H / D=0.61$, and the inner edge is $45^{\circ}$ chamfered to the height specified at $10 \mathrm{~mm}$, where $D$ and $H$ is the inner diameter and height, respectively. The inner diameter of a tangential nozzle is $d=3.2 \mathrm{~mm}$, and the height of the central area of the nozzle is $4 \mathrm{~mm}$ measured from the bottom of the cup. In order to observe pressure fluctuations, the workpiece was set at $\delta=4 \mathrm{~mm}$ [13]. The diameter, $D_{c}$, and height, $H_{c}$, of the column are made dimensionless providing the variation as follow; $H_{c} / H=0.34,0.57$, $0.90, D_{c} / \mathrm{D}=0.42,0.55,0.63,0.69,0.76$. The rotating coordinate system is placed at the center of the cup and at the same height of the nozzle. The vertical direction, the radius direction and the rotational angle are defined as $z$-axis, $r$-axis and $\theta$-axis. The rotational angle corresponds to the $r$-axis normal to the nozzle direction is $\theta=0^{\circ}$.

The cup was experimented under the Reynolds number $(\operatorname{Re}=V D / v$, here $V$ is swirling velocity, $v$ is kinematic viscosity) of 58,000 based on the air flow velocity near the wall [12] [13]. In this study, $V_{i n}$, the flow speed at the nozzle, was set at $3.4 \mathrm{~m} / \mathrm{s}$ to establish the flow similarity between the air-flow cup and the water-flow enlarged cup. In this case, $V_{w}$, the flow speed near the cup wall measured at same height of the nozzle, was $0.81 \mathrm{~m} / \mathrm{s}$, and the flow rate through the nozzle was $Q=27.3 \times 10^{-6} \mathrm{~m}^{3} / \mathrm{s}$. In this experiment, the supplying power does not change when the column is set in the chamber.

The dynamic pressure inside the cup was measured with $1 \mathrm{kHz}$ sampling rate via Validyne DP-15 straingauge transducer. Pressure taps were made at the central area of a workpiece and on the cup wall $(z / H=0.50, \theta$ $=210^{\circ}$ ). The measurement by particle image velocimetry (PIV) clarified the swirling flow condition beneath the workpiece. In order to get a better visualization of the cup, the cup was made by a transparent acrylic and set at the bottom of a test section tank such that an opened-end of the cup faced upward. A high speed camera (Photron, FASTCAM-SA1.1) was set above the test section tank. The capturing frame rate was $2000 \mathrm{fps}$, and the shutter speed was $1 / 20,000 \mathrm{sec}$. The CW-YAG Laser $(532 \mathrm{~nm}, 3 \mathrm{~W})$ provided a $2 \mathrm{~mm}$ laser light sheet illuminated the cup under the workpiece. Tracer particles, nylon 12 powder (mean diameter $150 \mu \mathrm{m}$, specific gravity 1.02) were used, and supplied far upstream of the nozzle.

\section{Experimental Results and Discussion}

Figure 5(a) shows the pressure signal without a column, and Figure 5(b) shows the spectrum analysis of the pressure signal shown in Figure 5(a). The fluctuation amplitude on the wall was obviously larger than that observed at the center of a work. The spectrum analysis extracted the frequency less than $10 \mathrm{~Hz}$. The dominant frequency was observed around $1 \mathrm{~Hz}$. It would have occurred from the fluctuation of the swirling flow.

Figure 6(a) shows the pressure signals with various columns, and Figure 6(b) shows the results of spectrum analysis of the pressure signals shown in Figure 6(a). It is noticed, as presented in Figure 5(a) and Figure 6(a), that without a column and with smaller diameter columns, the pressure fluctuation was not suppressed; but it was so with larger diameter columns. Based on this finding, the most important factor for suppression of the pressure fluctuation is the variation of the column diameter; the larger the better, while the height variation does not show significant effect to suppression of pressure fluctuation in the cup.

Figure 7 shows the amplitude of $p_{\text {rms }}$ which represents the pressure fluctuation. On the cup wall the $p_{\text {rms }}$ gradually decreased as the diameter increased. The $p_{\text {rms }}$ at the work center sharply decreased when $D_{c} / D$ reached 0.76. On the other hand, $H_{c} / H$ showed minimal influence on the suppression of the pressure fluctuation. It is concluded that the pressure fluctuation is suppressed when larger diameter column is presented.

In order to research the reason that pressure fluctuation is suppressed, the time sequence of the swirling flow

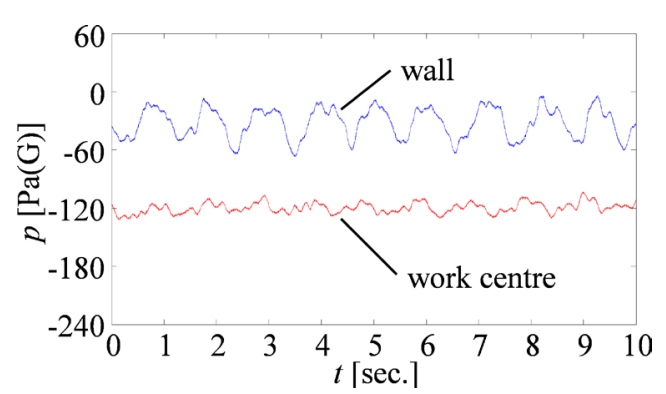

(a)

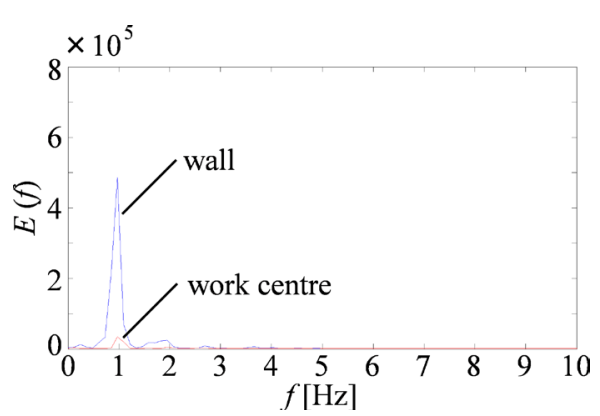

(b)

Figure 5. Pressure measurement results without a column. (a) Pressure signal; (b) Spectrum of pressure fluctuation. 


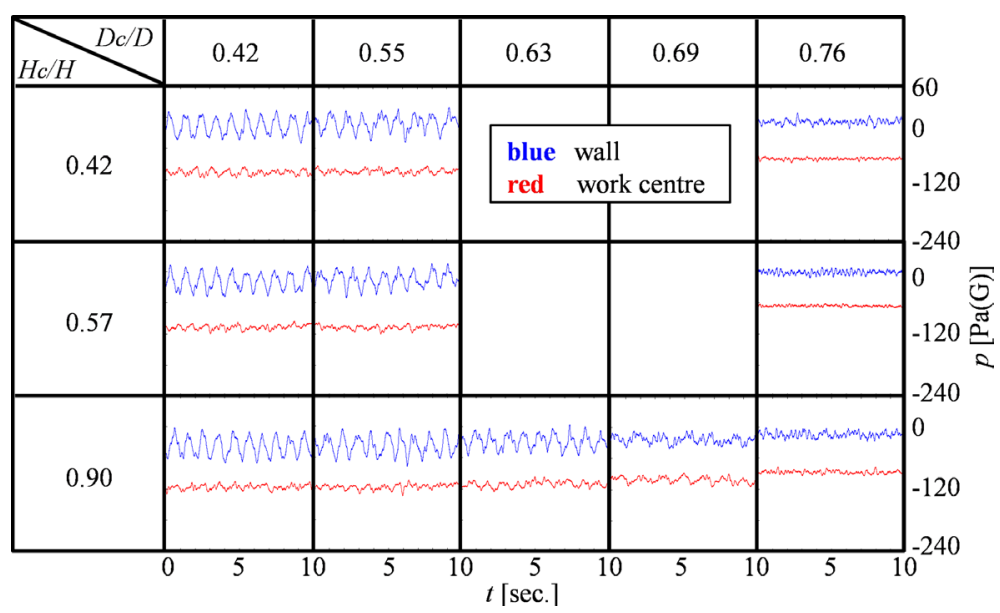

(a)

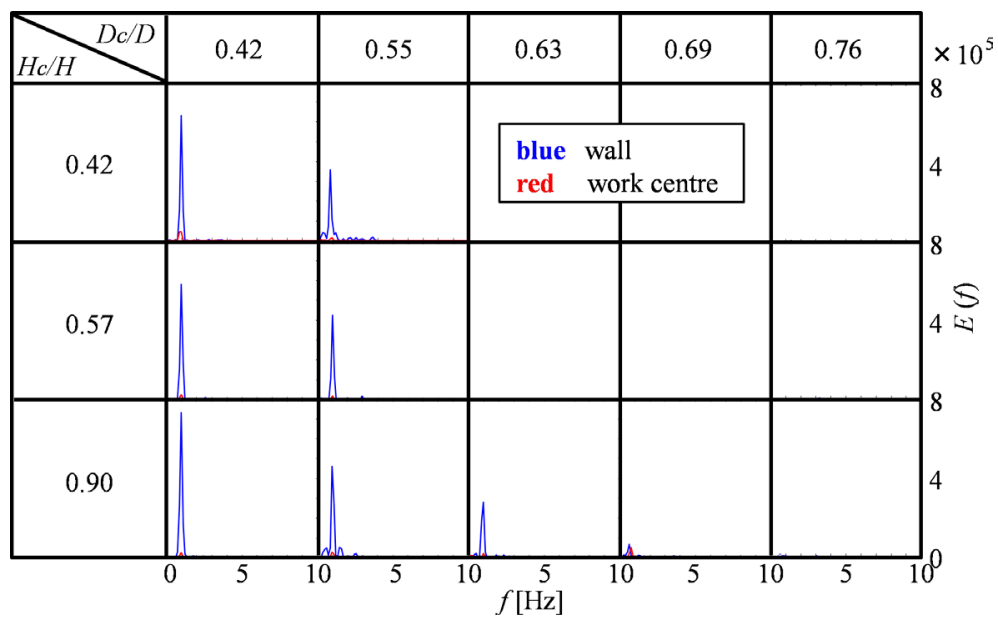

(b)

Figure 6. Pressure measurement results with various columns. (a) Pressure signal; (b) Spectrum of pressure fluctuation.

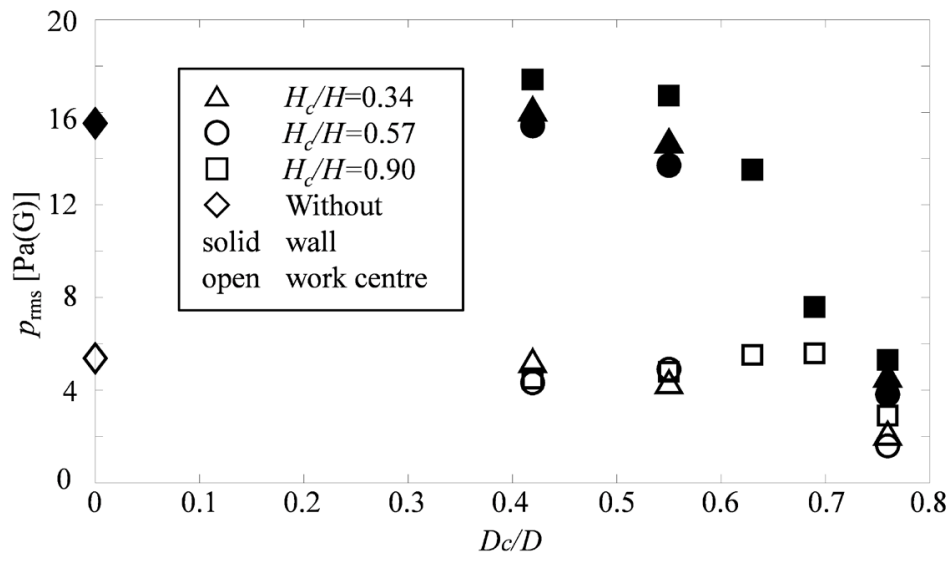

Figure 7. Pressure amplitude variation with various columns.

on the $r-\theta$ plane at $z=42 \mathrm{~mm}$ under the workpiece was focused. Figure 8 shows the instantaneous streamline which is drawn based on the PIV measurement result. The yellow circle, line and dot represent the inner cup wall, nozzle and the central area of the swirling flow, respectively. The swirl center was recognized roughly from high-speed images with eyes. It can be seen that, without a column, the swirling flow precessed, but when 
the large diameter column presented, the swirling flow did not precess. It is recognized that the swirling motion is more unstable from the comparison of streamline patterns with and without the column. It could be explained that when a larger diameter column is presented, the gap between the column wall and the cup wall become smaller. Therefore, the swirling flow fluctuation is suppressed such that the swirling flow under the workpiece becomes stable. The precession causes the pressure fluctuation at the work surface, which can be controlled by a column of selective diameter; the larger the diameter, the better the fluctuation suppression. Also, it has been found that larger diameter column brings an advantage to the vortex levitation.

Figure 9 shows the averaged streamline patterns of the swirling flow and the swirling center with variation of the column diameter. The position of a swirling center was identified by eye from the streamline pattern. Without a column, the streamline loses its pattern, but the pattern is maintained when a larger diameter column is presented. However, the large diameter column has a risk over the vortex levitation, too. As shown in Figure 10, the mean pressure, $p_{\text {mean }}$, increases of approximately $20 \%$ maximum as the diameter increases. When $D_{c} / D$ is smaller than $0.55, p_{\text {mean }}$ seems almost constant, but when $D_{c} / D$ is larger than $0.55, p_{\text {mean }}$ increases. The sucking force generated by a negative pressure becomes a little weakened.

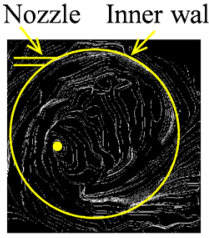

without column $t=0[\mathrm{sec}$.

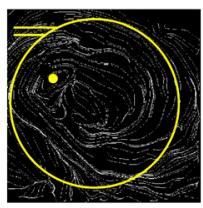

$t=0.5[\mathrm{sec}$.

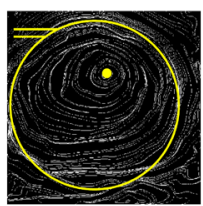

$D_{c} / D=0.76$

$t=0$ [sec.]

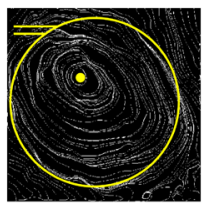

$t=0.5$ [sec.]

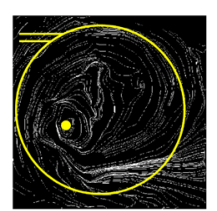

$t=0.1$ [sec.]

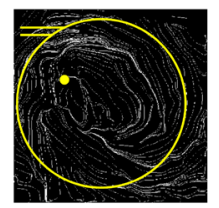

$t=0.6$ [sec.]

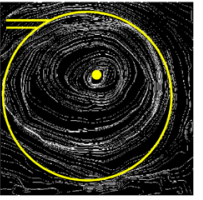

$t=0.1$ [sec.]

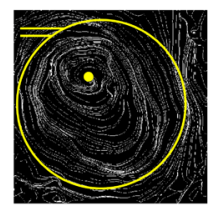

$t=0.6$ [sec.]

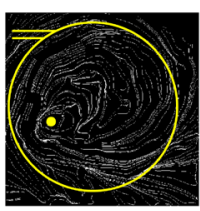

$t=0.2$ [sec.]

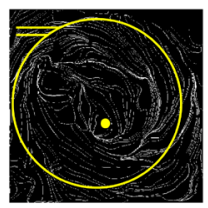

$t=0.7$ [sec.]

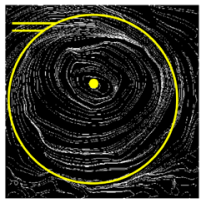

$t=0.2$ [sec.]

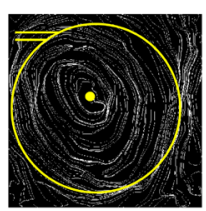

$t=0.7$ [sec.]

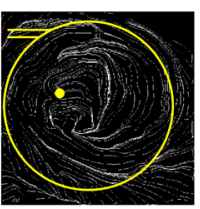

$t=0.3$ [sec.]

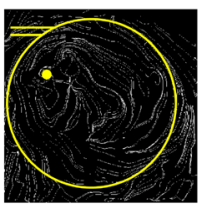

$t=0.8$ [sec.]

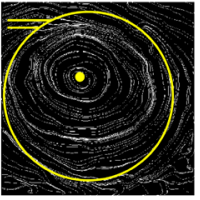

$t=0.3$ [sec.]

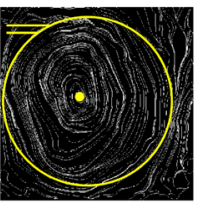

$t=0.8$ [sec.]

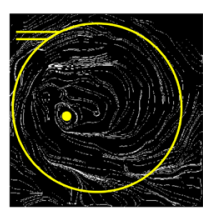

$t=0.4$ [sec.]

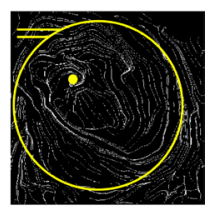

$t=0.9$ [sec.]

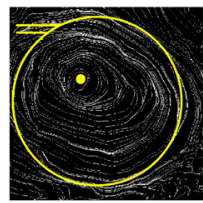

$t=0.4$ [sec.]

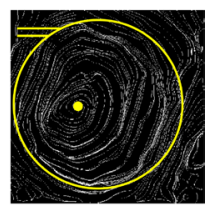

$t=0.9$ [sec.]

Figure 8. Time sequence motion of swirling flow core and instantaneous streamline.

Nozzle Inner wall

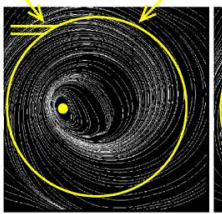

without

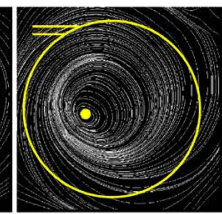

$D c / D=0.42$

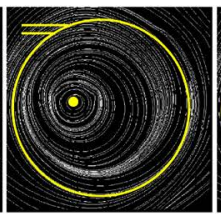

$D c / D=0.55$

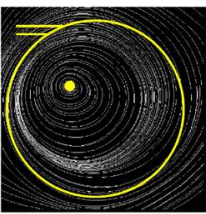

$D_{c} / D=0.63$

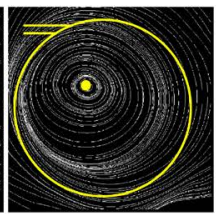

$D c / D=0.69$

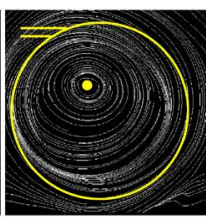

$D c / D=0.76$

Figure 9. Comparison of time averaged streamline pattern. 


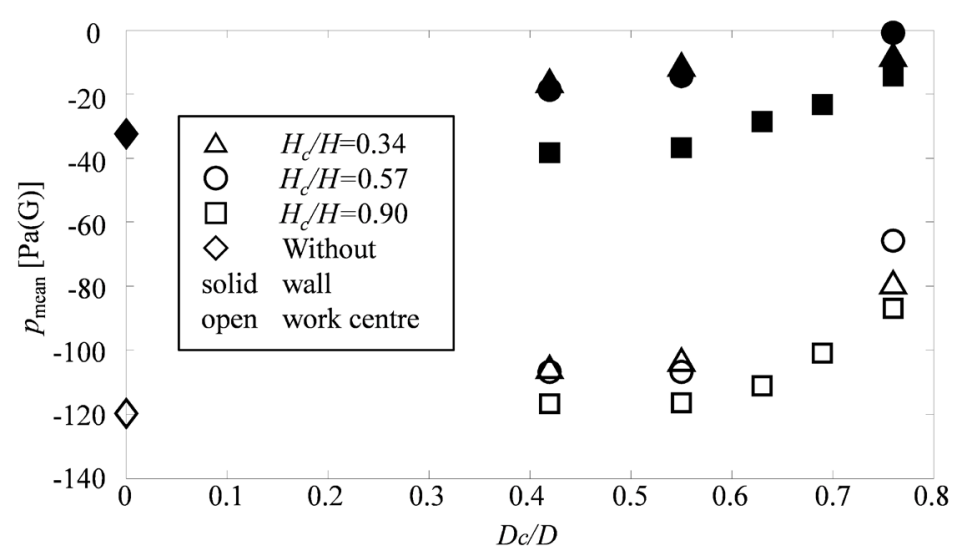

Figure 10. Mean pressure with various columns.

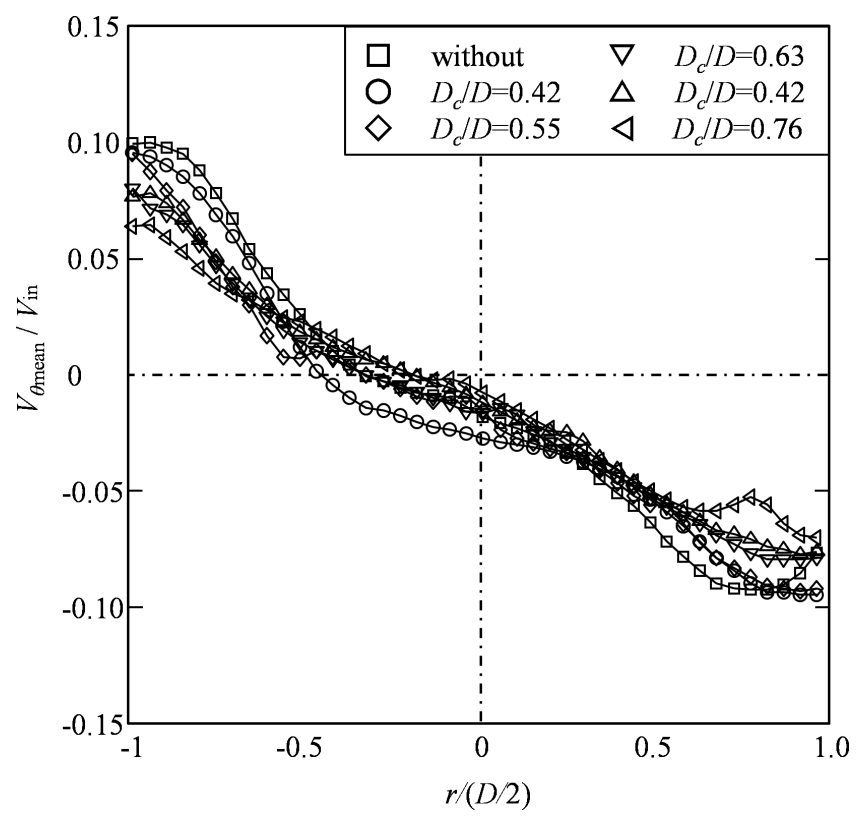

Figure 11. Distribution of swirl velocity.

In order to reveal the reason why the sucking force is weakened, $V_{\theta \text {,mean }}$ of the swirling flow under the workpiece on the $r \theta$-plane at $z / w=0.98$ was investigated. Figure 11 shows distribution of $V_{\theta \text {,mean }}$ on the line which parallel to the $x$-axis and through the swirl center. It has been found that, $V_{\theta \text {,mean }} / V_{i n}$ near \pm 1 becomes slower as the column becomes larger. The reason why the vacuum pressure is weakened is the decrease of the swirling velocity. It is considered that the drop of swirling velocity is related to the increasing wall friction.

From the afore mentioned results, it is possible to conclude that a larger diameter column can stabilize the non-contact handling device because it suppresses the pressure fluctuation, but the vacuum pressure becomes weakened according to a decrease in swirling velocity.

\section{Conclusion}

This study focused on the suppression of pressure fluctuation in the vortex cup by setting a cylindrical column in the cup chamber. Pressure measurement and flow visualization in the cup were conducted. As a result, the pressure fluctuation can be controlled with a larger diameter column, because of suppression of the swirling flow precessing. However, the height of the column does not affect the suppression. This is because the flowing direction between the column and the chamber is restricted circumferentially. In case of too large column, the swirling velocity weakens due to the shear stress effect from the small radial gap. 


\section{References}

[1] Brandt, E.H. (1989) Levitation in Physic. Science, 243, 349-355. http://dx.doi.org/10.1126/science.243.4889.349

[2] Vandaele, V., Lambert, P. and Delchambre, A. (2005) Non-Contact Handling in Microassembly (Acoustical Levitation). Precision Engineering, 29, 491-505. http://dx.doi.org/10.1016/j.precisioneng.2005.03.003

[3] Davis, S., Gray, J.O. and Caldwell, D.G. (2008) An End Effector Based on the Bernoulli Principle for Handling Sliced Fruit and Vegetables. Robotics and Computer-Integrated Manufacturing, 24, 249-257. http://dx.doi.org/10.1016/j.rcim.2006.11.002

[4] Dini, G., Fantoni, G. and Failli, F. (1995) Grasping Leather Plies by Bernoulli Grippers. CIRP Annals-Manufacturing Technology, 58, 21-24. http://dx.doi.org/10.1016/j.cirp.2009.03.076

[5] Waltham, C., Bendall, S. and Kotlicki, A. (2003) Bernoulli Levitation. American Journal of Physics, 71, 176-179. http://dx.doi.org/10.1119/1.1524162

[6] Ito, S., Ogawa, K. and Kuroda, C. (1979) Decay Process of Swirling Flow in a Pipe. International Journal of Chemical Engineering, 19, 600-605.

[7] Kitoh, O. (1991) Experimental Study of Turbulent Swirling Flow in a Straight Pipe. Journal of Fluid Mechanics, 225, 445-479. http://dx.doi.org/10.1017/S0022112091002124

[8] Nissan, A.H. and Bresan, V.P. (1961) Swirling Flow in Cylinders. AIChE Journal, 7, 543-547. http://dx.doi.org/10.1002/aic.690070404

[9] Cortes, C. and Gil, A. (2007) Modeling the Gas and Particle Flow inside Cyclone Separators. Progress in Energy and Combustion Science, 33, 409-452. http://dx.doi.org/10.1016/j.pecs.2007.02.001

[10] Kumar, R. and Conover, T. (1993) Flow Visualization Studies of a Swirling Flow in a Cylinder. Experimental Thermal and Fluid Science, 7, 254-262. http://dx.doi.org/10.1016/0894-1777(93)90009-8

[11] Nishimura, K., Takama, N., Endoh, T. and Yoshiki, H. (1990) Velocity Distribution of Swirl Flow in a Circular Cylinder. Manufacture Research, 42, 653-656.

[12] Iio, S., Umebachi, M., Li, X., Kagawa, T. and Ikeda, T. (2008) Research on a Non-Contact Handling System Using Swirling Flow (Influence of Cup-to-Work Gap Thickness on Swirling Flow Characteristics). SICE Transactions on Industrial Application, 7, 79-84.

[13] Iio, S., Umebachi, M., Li, X., Kagawa T. and Ikeda, T. (2010) Performance of a Non-Contact Handling Device Using Swirling Flow with Various Gap Height. Journal of Visualization, 13, 319-326. http://dx.doi.org/10.1007/s12650-010-0045-y

[14] Li, X., Iio, S., Kawashima, K. and Kagawa, T. (2011) Computational Fluid Dynamics Study of a Noncontact Handling Device Using Air-Swirling Flow. Journal of Engineering Mechanics, 137, 400-409. http://dx.doi.org/10.1061/(ASCE)EM.1943-7889.0000237

[15] Hayashi, K., Iio, S., Li, X. and Kagawa, T. (2013) Control of Swirling Flow Perturbation in a Noncontact Handling Device with a Circular Cylinder. Proceedings of the 12th International Symposium Fluid Control, Measurement and Visualization, Nara, 22 November 2013, 1-9.

[16] Li, X., Horie, M. and Kagawa, T. (2014) Pressure-Distribution Methods for Estimating Lifting Force of Swirl Gripper. IEEE/ASME Transactions on Mechatronics, 19, 707-718. 


\section{Nomenclature}

$D$ : Inner diameter of a vortex cup [mm]

$d$ : Diameter of the nozzle [mm]

$H$ : Height of a vortex cup [mm]

$p$ : Differential pressure between the cup and ambient $[\mathrm{Pa}]$

$Q$ : Flow rate $\left[\mathrm{m}^{3} / \mathrm{s}\right]$

$R e$ : Reynolds number $(=V D / v)$

$r$ : Radius position [mm]

$V$ : Velocity $[\mathrm{m} / \mathrm{s}]$

$z$ : Height position [mm]

$\delta$ : Gap height between a cup and a work piece [mm]

$\theta$ : Rotation angle [deg.]

\section{Subscript}

\section{$c$ : Column}

in: Issuing flow from a nozzle

mean: Averaged value

$r$ : Radial direction

rms: Root mean squared value

$w$ : Value near the wall of a cup

$z$ : z-direction

$\theta$ : Circumferential direction

$v$ : Kinematic viscosity 